\section{Possibility of estimating radioactive fallout by modelling atmospheric}

\section{processes}

\author{
HG Hasanov* and IM Zeynalov \\ Azerbaijan National Academy of Sciences, Department of Earth Sciences, Azerbaijan
}

Received: 27 March, 2020

Accepted: 21 April, 2020

Published: 22 April, 2020

*Corresponding author: HG Hasanov, Azerbaijan National Academy of Sciences, Department of Earth Sciences, Azerbaijan, E-mail: hhassanov@yahoo.com

Keywords: Radiation precipitation; Environmental protection; Meteorology; Climate-forming factors; Low-orbit satellites

https://www.peertechz.com

\section{Check for updates}

The paper suggests methods and means for solving problems of determining contamination by radioactive waste, appearing as precipitation when moving radioactive particles in the atmosphere. The model for predicting and evaluating radioactive fallout is developed. Meteorological conditions determine the conditions for turbulent diffusion of pollution on a regional and global scale.

The deposition of radioactive wastes like precipitation is formed as a result of the settling of long-lived explosion products from the atmosphere. If the parameters of the explosion (intensity, geometry, type of explosion and etc.) form a qualitative composition of radioactive products, then the effect of the meteorological influence are finally reduced to transport and change of the concentration of radioactive contamination.

Scattering of radioactive impurities is determined by stratification, turbulence and other parameters of the atmosphere, the direction and speed of their spatial distribution - the parameters of the direction and speed of the wind. Particles with sizes less than 10-12 microns moving at a speed identical to that of vertical movements $(\approx 1 \mathrm{~cm} / \mathrm{s})$ precipitate on the Earth's surface either in the turbulent motion of air masses (dry deposition), or by washing out sediments (wet and wet sediments) [1].

Assuming, in accordance with the well-known idea of Taylor and Schmidt [2], that the process of turbulent diffusion is equivalent to the process of molecular diffusion, one can obtain the next formula for the vertical turbulent heat flux in the surface layer of air.

$$
\mathrm{p}=p C p D\left(\theta_{w}-\theta\right)
$$

where $\rho$ is the air density (typical value is around $1.23 \times$ $\left.10^{-3} \mathrm{~g} / \mathrm{cm}^{3}\right), \mathrm{Cp}$ is the heat capacity of air at constant pressure (normally depends very strongly on physical conditions at which the air exists, and equal to $1,007 \mathrm{~kJ} / \mathrm{kg} \cdot \mathrm{K}$ at $300 \mathrm{~K}$ and dry weather), $\theta_{\mathrm{w}}$ is the temperature of the active Earth's surface, $\theta$ is the air temperature at a certain height, (measured in $\mathrm{m}$ ), $\mathrm{D}$ is the integral characteristic of the conditions of vertical turbulent transport between the underlying surface and the atmosphere $\left(\mathrm{cm}^{2} / \mathrm{sec}\right)$ which is called by M.I. Budyko [3] as the coefficient of external diffusion and is expressed in the form

$$
D=\frac{1}{\int_{0}^{\frac{z d z}{k}}}
$$

herein $\mathrm{z}$ is the air level at temperature $\theta$ (for example, if $\theta_{200 \mathrm{~cm}}$, then $\left.\mathrm{z}_{200 \mathrm{~cm}}\right), \mathrm{k}$ is the coefficient of turbulent exchange expressed in $\mathrm{cm}^{2} / \mathrm{sec}$ (Figure 1)'.

$$
\left(\frac{D_{i}}{V_{2 m}}\right)^{I, V I I}\left(\frac{\Delta \theta}{V_{2 M}^{2}}\right)^{I, V I I}
$$

Figure 1. Graph of the relationship between the climatic parameters of the stability parameter and the ratio of the integral turbulent exchange rate coefficient to the wind speed for the Transcaucasus region (black dots for July, bright for January) [3].

Crucial point herein is the character of the relationship between the integral coefficient of intensity of heat and 


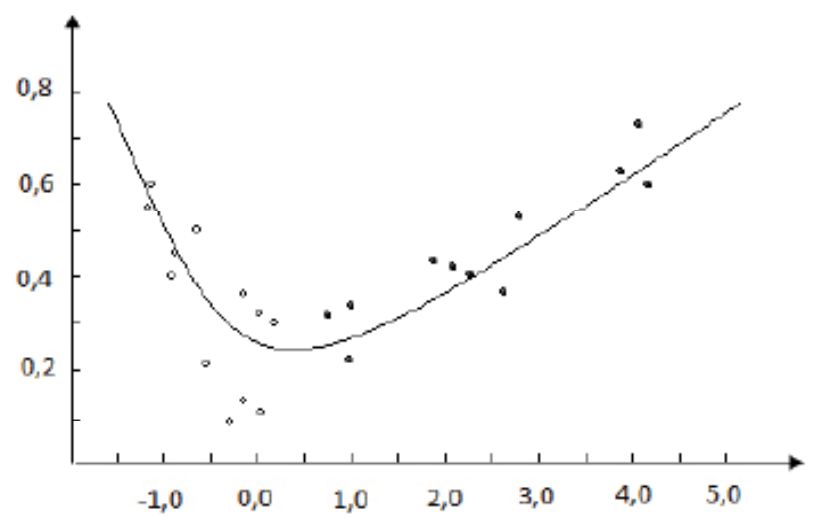

Figure 1:

moisture exchange (D) and the coefficient of turbulent exchange (k). In accordance with equation (2), we can write

$$
\int_{0}^{z \frac{d z}{k}}=D^{-1}
$$

Due to varying the value $\mathrm{D}$ between 0.5 and 1.3 in dependence on the climatic and landscape conditions, the average monthly values for climatological calculations will also have different and specific values which obey the same pattern [3].

Radioactive contamination of the terrain, among other things, depends on the speed of movement of radioactive particles in the atmosphere under the influence of wind. If we assume that the process of moving radioactive particles in the atmosphere is described by the diffusion equation, then for an excess concentration of particles marked as $\Delta \mathrm{n}$ (excess over the atmosphere, which is also radioactive), we have

$$
v_{z} \frac{d(\Delta n)}{d z}=\lambda \frac{d^{2}(\Delta n)}{d z^{2}}-\alpha\left(\Delta n-\Delta n_{0}\right.
$$

The following symbols are introduced into the equation (4): $\mathrm{v}_{\mathrm{z}}$ is the speed of the forced motion of radionuclides (in terms of the diffusion equation, this is the speed of the convection), and $\lambda$ is the coefficient of molecular diffusion of radionuclides in the layer under consideration.

When modeling this process, we assume that the excess concentration appeared as a result of a one-time radioactive explosion (this could be an explosion at a Nuclear Power Plant abbreviated below as NPP which is immediately blocked). The equation (4) describes the stationary distribution of radioactive particles as they move away from the nuclear power plant (from the epicenter of the explosion). For simplification of the approach an one-dimensional problem is considered when radionuclides move only in one direction (for example, in a plane layer of the atmosphere). An one-dimensional problem is considered when radionuclides move only in one direction (for example, in a plane layer of the atmosphere).

The last term in Eq. (4) describes the mass exchange between radionuclides emitted into the atmosphere and adjacent uncontaminated layers (the index $\alpha$ is the mass transfer coefficient); $\Delta \mathrm{n}_{0}-$ is exactly the "clean" or atmospheric concentration of particles (it is believed that this concentration is constant along the direction of movement of the radioactive cloud).

If there is a movement of radionuclides in several parallel (by altitude) layers, then the equation (4) can be generalized to the case of $\mathrm{n}$ set of meteorological and physical parameters, namely

$$
\sum_{i}^{n}=1 v_{z}^{(i) \frac{d(\Delta n) i}{d z}=} \sum_{i}^{n}=1 \lambda_{i}^{\frac{d^{2}(\Delta n) i}{d z^{2}}=} \sum_{i}^{n}=1 \alpha_{i}\left(\Delta n_{i}-\Delta n_{0 i}\right)
$$

The solution of the equation (5) is well known. Neglecting one of the solutions that will give rise physically incorrect result, we obtain the following expression for the excess concentration of radioactive particles in the atmosphere (more detailed see in [4])

$$
\Delta n=\Delta n_{0}+c \cdot \exp \left\{\left(\frac{v_{z}}{\lambda}-\sqrt{\Delta}\right) z / 2\right\}
$$

where $\Delta$ is the coefficient that determines the intensity of deposition of radioactive fallout $(\mathrm{RF})$ and expressed through meteo- and physical parameters of the atmosphere in the form

$$
\Delta=\left(\frac{v_{z}}{\lambda}\right)^{2}+4 \frac{\alpha}{\lambda}
$$

The analysis of the formula (7) implies a very simple conclusion: the higher the wind speed, the greater the distance from the emission source from which the radioactive particles will be carried away. In this sense, the most undesirable option for studied process is a constant by altitude wind both in direction, and in value. In this case, all radionuclides independently on their mass characteristics (chemical element, mass, isotope and etc.) will be concentrated in one place and in the form of RF fall onto the Earth's surface.

More preferable looks the option when the wind speed is different by altitude thereto the lower the altitude, the lower the wind speed. In this case, the zone of contamination of the terrain with heavy radionuclides will not be so wide, since heavy RF will fall down in areas near the source of pollution is located.

The zone of contamination with light radionuclides, on the contrary, will be more extensive. A priori, it will be very difficult to draw a conclusion about, when the total intensity of radiation contamination will be less or, conversely, more. It all depends on fact, which radionuclides were polluted into the atmosphere at the time of the explosion. The determination of the form of the function $\Delta \mathrm{n}(\mathrm{z})$ makes it possible to estimate the degree of contamination of the zones of interest to us by radionuclides.

Since within the framework of our model we assume that there are $n$ layers of radionuclides in the atmosphere, the total contamination per unit time and per unit length of the surface under consideration will be determined from the formula provided below. 


$$
N_{\text {summ }}=\sum_{i}^{n}={ }_{0} \lambda_{i} \frac{\partial(\Delta n) i}{\partial z}
$$

As a result of pollution of the RF on a regional and global scale, it is necessary to use ground-based and remote methods and means of observation. The most effective use of radar remote sensing systems (RSS) in conjunction with optoelectronic equipment $[5,6]$. The most benefit of radar RSS is the absence of weather and time (day/night) restrictions [7] for getting information.

Radar means for detecting radioactive meteorological formation of anthropogenic nature are very different (active and passive radar). Monitoring principles of nuclear cycle enterprises is based on the appearance of anthropogenic release in the lower layers of the atmosphere which leads to a change in the physical parameters of the propagation medium, which creates a radar contrast and makes it possible to detect atmospheric inhomogeneity.

Experimental values of specific effective scattering area (abbreviated as ESR) of radioactive emissions are due to:

a) Turbulent inhomogeneity of the air zone close to the exhaust pipe of NPP;

b) Increased density of particulates (water droplets, aerosols, clusters and so on) so that radioactivity contributes to the formation of clusters which, consequently, affect the growth of large drops;

c) Climatic and weather conditions, etc.

The analysis of the literature shows that for making studies targeted to creation of the satellite systems for the radar monitoring of radioactive releases it is necessary:

1. Creation of specialized multifrequency active and passive radars with increased energy potential;

2. Map of climatic and weather features of the area under study;

3. Data from meteorological satellites for comprehensive research;

4. Development of special algorithms for processing reflected radar signals [8].

\section{Conclusion}

In this paper the main factors of formation of radioactive wastes in the form of precipitation are revealed. On the basis of turbulent diffusion, a method has been demonstrated for the transport of contaminants, both regionally and globally.

A model for moving radionuclides in several parallel (altitudes) atmospheric layers is proposed. The model takes into account the meteorological parameters determining the intensity of radioactive waste deposition. The model can be applied to any territory and predict radioactive pollution of the territory; it's necessary just include into the model meteo - and terrain/landscape specifications of the territory under research.

\section{References}

1. Isaev AA (2001) Ecological Climatology. Textbook for the geographer Hydromet. Ekol. Special schools and colleges. Scientific world 458

2. Taylor GI (1922) Diffusion by continuous Movement. Proc Lond Math Soc 20 196-212.

3. Shikhlinsky EM (1969) Heat balance of the Azerbaijan SSR. Baku 200.

4. Hasanov HG (2002) Hydrodynamic research of interaction between acoustic and laser radiators with fluids (in Russian). Baku, Open World 384

5. Zeynalov IM (2005) Abstract for the degree of Candidate of Technical Sciences "Use of satellite data in the study of climatic characteristics of the territory of Azerbaijan Baku.

6. Zakharov $\mathrm{Al}$ (2012) Methods of remote sensing of the Earth by radars with synthesized aperture. Author's abstract. Diss. to the competition Ph.D. Moscow.

7. Nabiev Sh (2012) Modern trends in the development of methods for the remote detection of radioactive and highly toxic substances. Issled. Cente "Kurchatov Institute. Physics. Bulletin of the Russian Academy of Natural Sciences 14-26

8. Shilin BV, Gorny VI, Veremyev VI (2012) History of remote methods application in liquidation of the Chernobyl nuclear power plant accident" UDC 528.8 ISSN 2070-7401, Modern problems of remote sensing of Earth from space 9

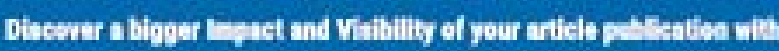
Peertechz Publleations

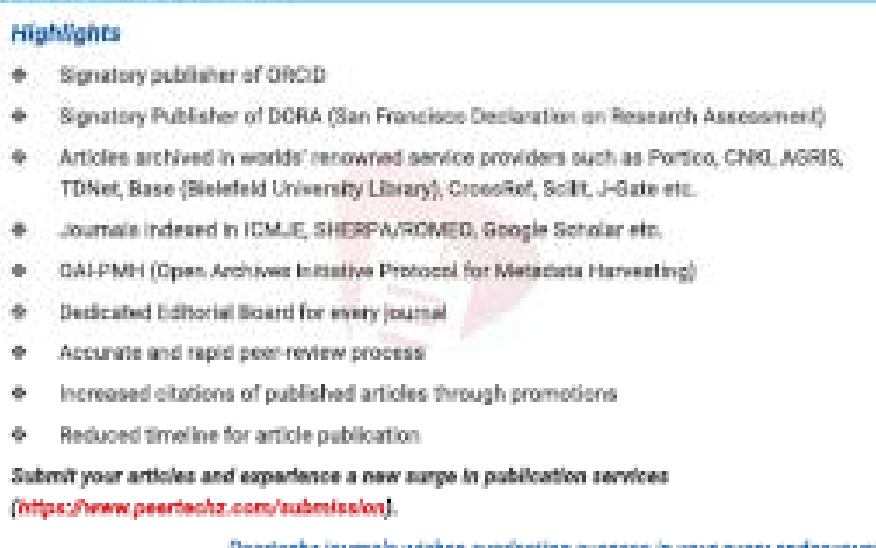

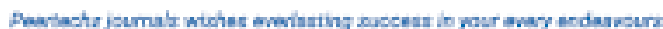

Copyright: ( 2020 Hasanov HG, et al. This is an open-access article distributed under the terms of the Creative Commons Attribution License, which permits unrestricted use, distribution, and reproduction in any medium, provided the original author and source are credited. 